\title{
Contaminación por cromo en el agua intersticial, en el agua del cauce y en los sedimentos del río Jarama
}

\author{
M. Arauzo*, M. Rivera*, M. Valladolid**, C. Noreña**, O. Cedenilla* \\ *Centro de Ciencias Medioambientales C.S.I.C.,Serrano 115 dpdo., 28006 Madrid, España. \\ ** Museo Nacional de Ciencias Naturales C.S.I.C.,José Gutiérrez Abascal 2, 28006 Madrid, España.
}

\section{RESUMEN}

Se ha realizado un estudio sobre la dispersión del cromo, en sus formas total y hexavalente, desde las aguas del cauce del río Jarama (Comunidad de Madrid, España) hacia otros compartimentos del ecosistema: medio intersticial, sedimentos y lagunas de gravera adyacentes al río. Las doce estaciones de muestreo estaban situadas en la zona media del Parque Regional del Sureste Madrid. Las concentraciones de cromo total en las aguas del río fueron superiores a las establecidas en los objetivos de calidad para aguas superficiales (Real Decreto 995/2000). El cromo se encontraba mayoritariamente en su forma hexavalente, mostrando concentraciones superiores, en un orden de magnitud, a las establecidas en los objetivos de calidad para este estado de valencia. Asimismo, los niveles de contaminación por cromo en los sedimentos y en el agua intersticial a orillas del cauce resultaron muy elevados, constituyendo importantes reservorios de cromo que podrían actuar como fuentes potenciales para la dispersión del mismo. Las lagunas de gravera adyacentes al río Jarama presentaron unas concentraciones de cromo en agua y sedimentos por debajo de los límites de calidad establecidos. Parece improbable, por tanto, que se hayan producido procesos de filtración del contaminante desde el río hacia las lagunas.

Además del proceso de transporte y acumulación de cromo en el ecosistema fluvial, otro ecosistema receptor del contaminante es el constituido por los campos de cultivo que reciben aguas de riego procedentes del río. La elevada concentración de cromo hexavalente en las aguas del Jarama entraña un importante riesgo para la salud humana debido, fundamentalmente, a su elevada carcinogenicidad por inhalación. Se desaconseja el riego por aspersión durante las prácticas agrícolas a fin de evitar la posible inhalación de aerosoles contaminados.

Palabras clave: cromo total, cromo hexavalente, agua intersticial, sedimento, río Jarama.

\begin{abstract}
The dispersion of chromium (total chromium and hexavalent chromium) from water of the River Jarama (Comunidad de Madrid, Spain) into the interstitial medium, into sediments and into adjacent gravel-pit lakes was studied. Twelve sampling stations were selected in the middle reaches of Parque Regional del Sureste de Madrid. Concentrations of total chromium in the water of the river were found to be higher than target concentrations for this chemical species in surface waters (Spanish Royal Decree no. 995/2000). Chromium was present in its hexavalent form, showing concentrations one order of magnitude higher than the quality standards for CrVI. Pollution levels in sediments and interstitial water in the river banks were very high as well, thus forming a reservoir of chromium which could potentially spread the contaminant. The probability of chromium leaking out of the river channel and into the adjacent gravel-pit lakes seems rather low, as the latter showed chromium concentrations -in water and sediments- well below the acceptable quality levels.

In addition to transport and accumulation of chromium in the fluvial ecosystem, nearby agricultural ecosystems are also badly affected by the contaminant. Due to its high carcinogenicity, the presence of hexavalent chromium in the River Jarama poses a major health risk. In particular, sprinkler irrigation should be avoided in farming in order to prevent inhalation of CrVI-polluted aerosols.
\end{abstract}

Keywords: total chromium, hexavalent chromium, interstitial water, sediment, River Jarama. 


\section{INTRODUCCIÓN}

El cromo es un elemento que ha sido identificado tanto como un micronutriente esencial, como un agente carcinogénico, dependiendo de su forma química (Katz \& Salem, 1994). En su aplicación industrial, forma parte de curtientes, pigmentos y conservantes textiles, aleaciones, pinturas antiincrustantes, catalizadores, agentes anticorrosivos, lodos de perforación, baterías de alta temperatura, fungicidas, conservantes de madera, recubrimientos metálicos y electrogalvanizados (Katz \& Salem, 1994; U.S.EPA, 1998). Una vez iniciado el proceso de acumulación de cromo en el medio ambiente a partir de distintas fuentes (naturales o antropogénicas), puede verse transferido de unos compartimentos a otros del ecosistema: aire, aguas superficiales, sedimentos, aguas subterráneas, suelos y seres vivos.

El vertido incontrolado de cromo al medio ambiente se genera, principalmente, porque la mayor parte de los sistemas colectores de aguas residuales no posibilitan la separación de efluentes urbanos e industriales, de modo que las aguas residuales de las zonas urbanas con polígonos industriales son siempre de tipo mixto. Los sistemas convencionales para depuración de aguas residuales urbanas (tratamiento primario + secundario) presentan unos rendimientos medios para la remoción del cromo (y de otros metales mesados) que distan mucho de poder ser considerados eficientes (U.S.EPA, 1992). Aunque se han desarrollado procesos tecnológicos específicos para la eliminación de los metales pesados de las aguas residuales (Palmer et al., 1988) su aplicación es altamente costosa y están muy poco generalizados. Estas limitaciones en el tratamiento conllevan, con frecuencia, el vertido de elevadas concentraciones de metales pesados, que se reparten a lo largo de la cuenca receptora y ecosistemas aledaños. El Real Decreto 995/2000 (BOE n ${ }^{\circ}$ 147, de 20 de junio de 2000), por el que se fijan los objetivos de calidad para determinadas sustancias contaminantes en las aguas superficiales continentales, incluye al cromo en la lista de sustancias preferentes y fija unas concentracio- nes límite de $50 \mu \mathrm{g} \mathrm{L}^{-1}$ para el cromo total y de $5 \mu \mathrm{g} \mathrm{L}^{-1}$ para el cromo hexavalente. W.A.EPA (1993) restringe los niveles permisibles de cromo total en aguas superficiales a $10 \mu \mathrm{g} \mathrm{L}-1$.

En el presente estudio se han evaluado los niveles de contaminación por cromo, en sus formas total y hexavalente, en el tramo medio del río Jarama y se ha determinado el grado de dispersión del contaminante hacia otros compartimentos del ecosistema. Para ello se han analizado diferentes ambientes, como el agua del cauce, el agua intersticial a orillas del cauce, los sedimentos del río y las lagunas de gravera adyacentes al río (salvo los contaminantes transportados por el propio río, no se han observado indicios sobre la existencia de otros posibles aportes de cromo, por vertidos industriales u otros orígenes, en los diversos ambientes estudiados). El trabajo se desarrolló siguiendo el curso del Jarama a su paso por Arganda del Rey, Rivas de Vaciamadrid y San Martín de la Vega, municipios que se sitúan en la zona media del Parque Regional del Sudeste de la Comunidad de Madrid (España). Arauzo et al. (2002) estimaron un vertido anual de $2.2 \mathrm{Tm}$ de cromo total desde el efluente de la depuradora de Arganda del Rey al río Jarama, del cual, el $63 \pm 21 \%$ era cromo hexavalente, resultados que apuntaban hacia el desarrollo de un claro proceso de contaminación por cromo en el área citada. El Jarama, con una cuenca hidrográfica de $11597 \mathrm{~km}^{2}$, es el más largo y caudalosos de los afluentes del río Tajo por su margen derecha. Atendiendo a la revisión bibliográfica que realizó Rovira (1993) sobre la calidad de sus aguas, el río Jarama puede dividirse en tres zonas: desde su nacimiento hasta su confluencia con el río Guadalix (agua de buena calidad, con buena oxigenación, $\mathrm{pH}$ de 5.5 a 7.7, los valores de los índices bióticos son elevados, la contaminación orgánica no sobrepasa la capacidad autodepuradora del río y la contaminación por metales pesados no es significativa), desde la desembocadura del río Guadalix hasta la del Manzanares (el oxígeno disminuye, la capacidad autodepuradora disminuye hasta perderse debido a los aportes urbanos e industriales y la diversidad de 
macroinvertebrados se reduce de forma alarmante) y desde la afluencia del río Manzanares hasta la desembocadura del Tajo (el caudal del Jarama aumenta por el aporte del Manzanares, constituido mayoritariamente por aguas residuales, los niveles de contaminación de origen urbano e industrial son muy elevados, desaparecen las comunidades de macroinvertebrados). El área de estudio del presente trabajo se situaría en las zonas segunda y tercera, con relación a la tipificación citada por Rovira.

La química medioambiental de los compuestos del cromo incluye procesos de oxidaciónreducción, reacciones de precipitación-solubilización y fenómenos de adsorción-desorción. En la naturaleza, aparecen mayoritariamente compuestos de cromo trivalente (Cr III) y de cromo hexavalente (Cr IV), aunque los primeros son los más abundantes. La solubilidad del Cr III está limitada por la formación de diversos tipos de óxidos e hidróxidos. También presenta una fuerte tendencia a formar complejos estables con especies orgánicas e inorgánicas cargadas negativamente. Debido a esto, el Cr III no migra de manera significativa en el rango de $\mathrm{pH}$ cubierto por las aguas naturales, sino que precipita rápidamente y se adsorbe en partículas en suspensión y sedimentos del fondo. Los compuestos de Cr VI, sin embargo, son solubles en un rango mayor de condiciones ambientales. En solución, el Cr VI existe en forma de hidrocromatos, cromatos y dicromatos, dependiendo del $\mathrm{pH}$; los cromatos predominan a $\mathrm{pH}$ básico y neutro, los hidrocromatos a $\mathrm{pH}$ ligeramente ácido (6 a 6.2), mientras que a $\mathrm{pH}$ muy bajo predominan los dicromatos (U.S.EPA, 1998). El cambio de un estado de valencia a otro depende de factores como la materia orgánica, el pH y el potencial redox. Agentes reductores naturales como el hierro (II), el azufre (II) y la materia orgánica pueden transformar los compuestos de Cr VI en compuestos de Cr III en el medio natural (Bartlett \& Kimble, 1976; Eary \& Rai, 1989, U.S.EPA, 1998), siendo la tasa de reducción dependiente del potencial redox y el $\mathrm{pH}$ (Saleh et al., 1989). En aguas aeróbicas, el Cr VI permanece estable en su forma disuelta. En medio ácido los compuestos de Cr VI se comportan como fuertes agentes oxidantes, de manera que es rápidamente reducido a $\mathrm{Cr}$ III. $\mathrm{Si}$ el $\mathrm{pH}$ es elevado, el Cr III se oxida fácilmente a Cr VI (Kazt y Salem, 1994).

Debido a la gran movilidad y la elevada toxicidad del Cr VI, su presencia por encima de los niveles recomendados (Real Decreto 995/2000) puede generar un grave riesgo para la salud pública. U.S. EPA (1998) elaboró una amplia revisión toxicológica sobre los efectos del Cr VI en la que se resalta su biodisponibilidad como el factor más determinante para que una fuente específica de cromo pueda resultar tóxica; estudios epidemiológicos demostraron su poder carcinogénico por inhalación, de ahí la importancia en el control de riesgos durante el riego por aspersión, el uso de torres de refrigeración, u otros sistemas que favorezcan la inhalación de aguas contaminadas por $\mathrm{Cr}$ VI.

En este trabajo se ha evaluado el cumplimiento de los estándares de calidad establecidos para el cromo total y el cromo hexavalente en el tramo medio del río Jarama. El estudio de diferentes compartimentos del ecosistema fluvial ha permitido determinar las posibles vías de dispersión del cromo, así como los puntos del ecosistema en los que se está produciendo un proceso de acumulación del contaminante.

\section{MATERIAL Y MÉTODOS}

La campaña de muestreos se desarrolló durante los días 21 y 22 de noviembre de 2001. Se seleccionaron 13 estaciones de muestreo, siguiendo el curso del río Jarama a su paso por Arganda del Rey, Rivas de Vaciamadrid y San Martín de la Vega (en la zona media del Parque Regional del Sudeste de la Comunidad de Madrid, España). En la Tabla 1 se hace una relación de las estaciones de muestreo y de los tipos de muestra recogidos. Se tomaron muestras de agua del cauce del río, de agua intersticial a orillas del cauce y de sedimento, tanto en el río Jarama, como en el Manzanares en su confluencia con el Jarama. También se recogie- 
Tabla 1. Estaciones de muestreo y tipos de muestra. Sampling stations and sample types.

\begin{tabular}{|c|c|c|c|c|c|c|}
\hline $\begin{array}{l}\text { Estación } \\
\left(n^{\circ}\right)\end{array}$ & Localización & Sistema & $\begin{array}{l}\text { Profundidad } \\
\text { total }(\mathrm{m})\end{array}$ & $\begin{array}{l}\text { Muestreo } \\
\text { de agua en } \\
\text { río/laguna }\end{array}$ & $\begin{array}{c}\text { Muestreo } \\
\text { de agua } \\
\text { intersticial }\end{array}$ & $\begin{array}{c}\text { Muestreo } \\
\text { de sedimento }\end{array}$ \\
\hline 1 & $\begin{array}{l}\text { Puente Antiguo del } \\
\text { Ferrocarril de Arganda }\end{array}$ & río Jarama & 2.50 & muestra integrada & - & sí \\
\hline 2 & $\begin{array}{l}\text { Depuradora de Arganda } \\
\text { (efluente) }\end{array}$ & río Jarama & - & muestra de superficie & - & sí \\
\hline 3 & Soto de El Rincón & río Jarama & - & muestra de superficie & sí & sí \\
\hline 4 & Puente de Arganda & río Jarama & 1.75 & muestra integrada & - & sí \\
\hline 6 & $\begin{array}{l}\text { Manzanares (junta } \\
\text { con el río Jarama) }\end{array}$ & río Manzanares & - & muestra de superficie & sí & sí \\
\hline 7 & $\begin{array}{l}\text { Jarama (junta con } \\
\text { el río Manzanares) }\end{array}$ & río Jarama & - & muestra de superficie & sí & sí \\
\hline 9 & Presa del Rey & río Jarama & 2.00 & muestra integrada & sí & sí \\
\hline 10 & Puente Pindoque & río Jarama & - & muestra de superficie & - & sí \\
\hline 12 & $\begin{array}{l}\text { Puente hacia San Martín } \\
\text { de la Vega }\end{array}$ & río Jarama & 1.00 & muestra integrada & sí & sí \\
\hline 5 & $\begin{array}{l}\text { Laguna de } \\
\text { El Campillo }\end{array}$ & laguna de gravera & 5.50 & muestra integrada & - & sí \\
\hline 8 & $\begin{array}{l}\text { Laguna de Soto } \\
\text { de las Juntas }\end{array}$ & laguna de gravera & 2.25 & muestra integrada & - & sí \\
\hline 11 & $\begin{array}{l}\text { Laguna de } \\
\text { Los Ángeles }\end{array}$ & laguna de gravera & 1.00 & muestra integrada & - & sí \\
\hline 13 & $\begin{array}{l}\text { Laguna de Soto } \\
\text { Pajares } 5\end{array}$ & laguna de gravera & 4.25 & muestra integrada & - & sí \\
\hline
\end{tabular}

ron muestras de agua y de sedimento en cuatro lagunas de gravera adyacentes al río Jarama. Debido a su gran proximidad al cauce fluvial y dado que las citadas lagunas son zonas de aliviadero del acuífero subyacente, se consideró que las calidades del agua y de los sedimentos de las lagunas podrían ser indicadores de la existencia de procesos de dispersión del cromo, por filtración desde el río, hacia las capas superiores de acuífero.

En las estaciones de muestreo con profundidad suficiente (Tabla 1) se realizaron perfiles in situ de temperatura, oxígeno disuelto, $\mathrm{pH}$ y conductividad, cada $0.5 \mathrm{~m}$ de profundidad. Asimismo, para su analítica posterior, se prepararon muestras de agua integradas, a partir de alícuotas recogidas cada $0.5 \mathrm{~m}$ (desde la superficie hasta el fondo). La extracción de muestras de agua a diferentes niveles de profundidad se realizó mediante el uso de una botella de muestreo tipo Van Dorn de apertura horizontal y $2.5 \mathrm{~L}$ de capacidad. En las estaciones fluviales más someras, la medición de las variables citadas y la recogida de muestras de agua se realizaron en superficie (Tabla 1).

La temperatura del agua y el oxígeno disuelto se midieron con un oxímetro de campo YSI57. Para la medida del $\mathrm{pH}$ y de la conductividad se utilizaron un pHmetro de campo HANNA HI 9023 y un coductivímetro de campo HANNA HI 9033.

Las muestras de agua intersticial se recogieron según el método de Karaman-Chappuis (Chappuis, 1942), excavando en zonas no inundadas, a una distancia de 1.5 a $2.0 \mathrm{~m}$ del cauce del río, hasta alcanzar el nivel en el que aparecía el agua del medio intersticial (hacia 0.50$0.70 \mathrm{~m}$ de profundidad).

En aquellas estaciones en las que se recogieron muestras de agua intersticial (puntos 3,6, 7,9 y 12) se determinó un parámetro adicional que denominamos "ángulo entre el litoral y el sentido de la corriente del río”. Este parámetro 
permitió evaluar la influencia de la morfología del cauce sobre la acumulación de contaminantes en las aguas del medio intersticial. En las zonas del litoral que oponen resistencia a la corriente del agua (recodos, pequeños meandros), donde el ángulo es mayor, se espera una concentración de contaminantes más elevada, debido al efecto de filtrado y acumulación en los intersticios entre arenas y gravas. En este caso, el cromo transportado por la corriente y forzado a penetrar en el talud, puede ser fácilmente adsorbido por la abundante presencia de limos y materia orgánica acumulados en estos lugares; también se facilita la sedimentación de las partículas en suspensión transportadas por el río, con cromo ya adsorbido. Cuando, sin embargo, la línea del litoral y el sentido de la corriente se disponen en paralelo, la resistencia es mínima y se espera menor presión de filtrado sobre los materiales del litoral.

El cromo hexavalente en las muestras de agua se analizó por colorimetría, mediante el método del difenil carbacida en solución ácida (APHA, 1998). Para la determinación del cromo total se utilizó la misma técnica analítica, previa oxidación de la muestra con permanganto potásico (a fin de convertir todo el cromo a su forma hexavalente). La demanda química de oxígeno (DQO) se evaluó mediante la técnica del dicromato potásico (APHA, 1998). El nitrógeno total y el fósforo total se determinaron por colorimetría, el primero mediante el método 2.6-dimetil fenol (Lange, 1998) y el segundo mediante el método del fosfomolibdeno (Murphy \& Riley, 1962). Todas las muestras de agua (tanto de superficie, como intersticial) se analizaron durante el mismo día de su recogida.

Las muestras de sedimento se extrajeron con una draga tipo Petersen. La analítica del cromo total sobre las muestras de sedimento se realizó mediante espectometría de emisión ICP-AES (plasma de acoplamiento inductivo), previa digestión mediante ataque nítrico-perclórico. Dada la heterogeneidad granulométrica de las muestras de sedimento recogidas, los resultados se refirieron a la fracción igual o inferior a 0.02 $\mathrm{mm}$, lo cual permitió que los resultados en todas las estaciones fueran comparables entre sí. Si bien es cierto que no existe un consenso acerca de la fracción de sedimento a utilizar, existe una mayor tendencia al uso partículas de pequeño tamaño que, por su elevada relación superficie-volumen, pueden tener muchos lugares de unión potenciales con los metales pesados (Förstner \& Wittmann, 1981; Förstner, 1983; Moriarty \& Hanson, 1988). Para la estimación de la concentración de cromo total en la fracción de sedimento con un tamaño de partícula igual o inferior a $0.02 \mathrm{~mm}$ se aplicó, previamente, el método de densimetría de Bouyoucos (1929), con la finalidad de determinar las fracciones granulométricas en cada muestra de sedimento; así, se obtuvieron las proporciones de arenas y gravas $(>0.02 \mathrm{~mm}) \mathrm{y}$ de limos y arcillas $(<0.02 \mathrm{~mm})$ y, posteriormente, se calculó la concentración de cromo correspondiente a la fracción fina de cada muestra, a partir del contenido de cromo total en la muestra completa de sedimento.

Se utilizó la técnica multiparamétrica de Análisis de Componentes Principales (StatSoft, Inc. Staff, 1995) para ordenar e interpretar los resultados obtenidos a partir de las muestras de agua de origen fluvial y de agua intersticial.

\section{RESULTADOS}

En la figura 1 se muestran las concentraciones de cromo total y de cromo hexavalente en las estaciones de los ríos Jarama y Manzanares y en las lagunas de gravera adyacentes al río Jarama. En las aguas del Jarama se observaron concentraciones de cromo total entre $0.05 \mathrm{y}$ $0.10 \mathrm{mg} \mathrm{L}^{-1}$. En el efluente de la depuradora de Arganda (estación 2) se registraron los niveles más elevados. Todas las estaciones del río Jarama previas a su confluencia con el Manzanares presentaron concentraciones de cromo total superiores a las establecidas en los objetivos de calidad para determinadas sustancias contaminantes en aguas superficiales (Real Decreto 995/2000). La proporción de Cr VI con respecto al cromo total era del $100 \%$ en la 


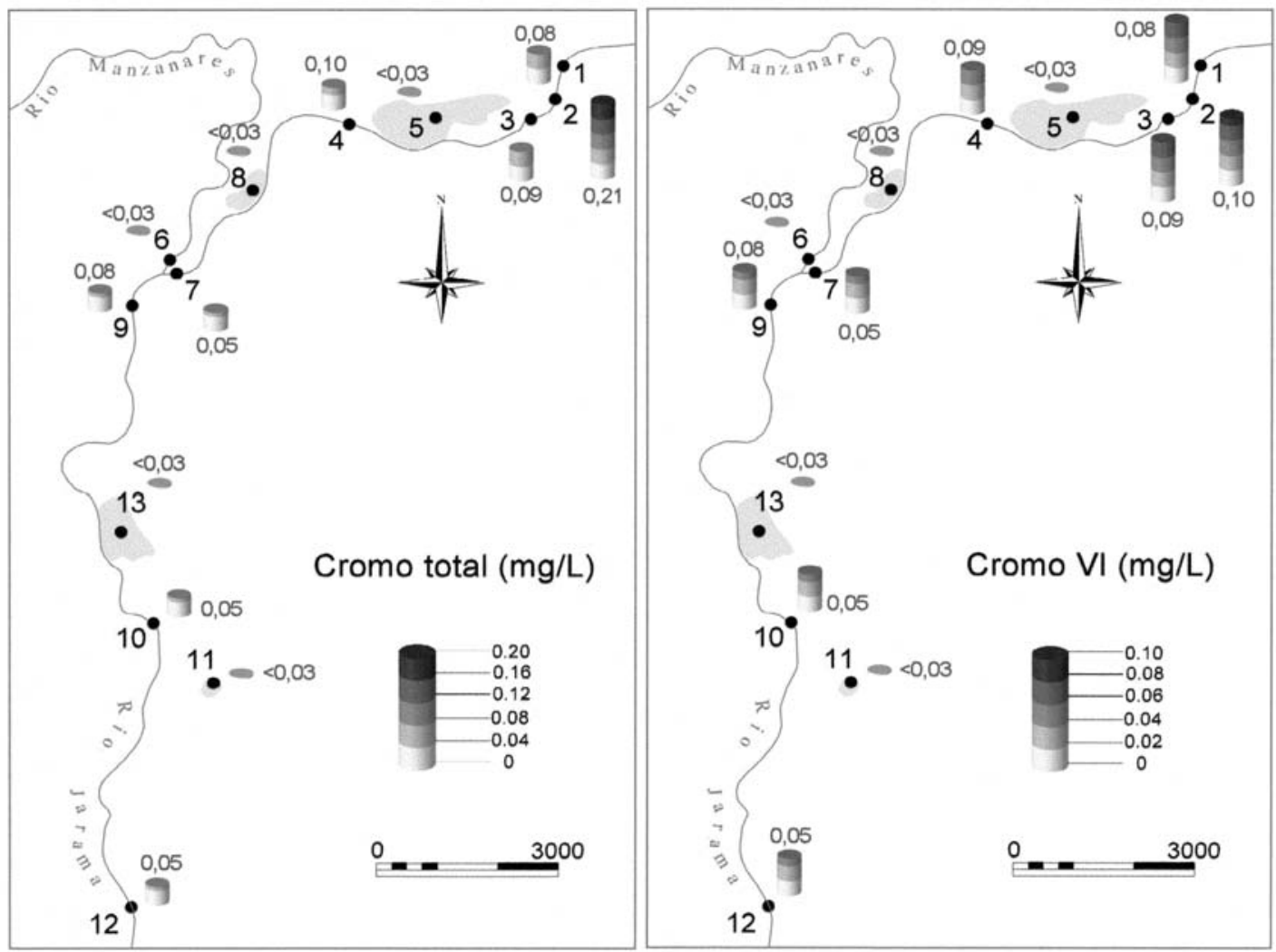

Figura 1 Concentraciones de cromo total y de cromo hexavalente en el agua de los ríos Jarama y Manzanares y en las lagunas de gravera. La numeración de las estaciones de muestreo corresponde a la descrita en la Tabla 1. Total chromium and hexavalent chromium concentrations in the water of the Rivers Jarama and Manzanares and the gravel-pit lakes. Numbering of the sampling stations is shown in Table 1.

mayor parte de las estaciones del Jarama, presentando concentraciones superiores, en uno y dos ordenes de magnitud, a los valores establecidos como los objetivos de calidad para aguas superficiales. El pH observado en el agua, neutro o ligeramente alcalino (Tabla 2), sugiere que la forma química predominante de Cr VI eran los cromatos (U.S.EPA, 1998). En el río Manzanares (estación 6) y en las cuatro lagunas de gravera (estaciones 5, 8, 11 y 13 ) las concentraciones de cromo total y $\mathrm{Cr}$ VI se encontraron por debajo de los límites establecidos como objetivos de calidad.

La temperatura, el $\mathrm{pH}$, la conductividad, el oxígeno disuelto, el fósforo total, el nitrógeno total y la DQO en el agua de las 13 estaciones se muestran en la Tabla 2. Las cuatro lagunas de gravera aparecían mezcladas en toda su profundidad. El pH mostró valores levemente alcalinos en todas las estaciones, aunque en las lagunas era ligeramente superior. La conductividad no presentó gran variabilidad entre las diversas estaciones fluviales, si bien, en el efluente de la depuradora y en las cuatro lagunas se dieron valores más elevados. Se observaron concentraciones de oxígeno disuelto entre 1 y $8 \mathrm{mg} \mathrm{L}^{-1}$. La estación del río Manzanares (punto 6), presentó los niveles más bajos de oxígeno (en concordancia con la gran carga de materia orgánica que transporta el río en esa zona). Se observaron valores muy elevados de DQO, nitrógeno total y fósforo total en todas las estaciones de muestreo, registrándose los niveles más altos en el efluente de la depuradora y en el río 
Tabla 2. Temperatura, oxígeno disuelto, $\mathrm{pH}$, conductividad, fósforo total, nitrógeno total y DQO en las muestras de agua procedentes del cauce de los ríos Jarama y Manzanares y de las lagunas de gravera adyacentes al Jarama. En las estaciones en las que se realizaron perfiles, se presenta el valor medio \pm la desviación estándar en la columna de agua. Temperature, dissolved oxygen, pH, conductivity, total phosphorous, total nitrogen and COD in the water samples from the Rivers Jarama and Manzanares and the gravel-pit lakes adjacent to the River Jarama. Mean value \pm standard deviation in the water column is shown for stations where deep profiles were performed.

\begin{tabular}{|c|c|c|c|c|c|c|c|c|}
\hline $\begin{array}{l}\text { Estación } \\
\left(\mathbf{n}^{0}\right)\end{array}$ & Localización & $\begin{array}{l}\text { Tra. } \\
\left({ }^{\circ} \mathrm{C}\right)\end{array}$ & $\underset{\left(\mathrm{mgL}^{-1}\right)}{\mathrm{O}_{2}}$ & $\mathrm{pH}$ & $\begin{array}{c}\text { Cond. } \\
\left(\mu \mathrm{Scm}^{-1}\right)\end{array}$ & $\begin{array}{c}\text { PT } \\
\left(\mathrm{mgL}^{-1}\right)\end{array}$ & $\begin{array}{c}\mathrm{NT} \\
\left(\mathrm{mgL}^{-1}\right)\end{array}$ & $\begin{array}{l}\text { DQO } \\
\left(\mathrm{mgL}^{-1}\right)\end{array}$ \\
\hline 1 & $\begin{array}{l}\text { Puente Antiguo } \\
\text { del Ferrocarril } \\
\text { de Arganda }\end{array}$ & $13.7 \pm 0.1$ & $7.8 \pm 0.3$ & $7.8 \pm 0.0$ & $939 \pm 11$ & 1.40 & 13.8 & 31 \\
\hline 2 & $\begin{array}{c}\text { Depuradora de Arganda } \\
\text { (efluente) }\end{array}$ & 14.5 & 5.0 & 7.6 & 1521 & 1.50 & 20.0 & 98 \\
\hline 3 & Soto de El Rincón & 10.1 & 5.4 & 7.7 & 986 & 1.60 & 14.0 & 28 \\
\hline 4 & Puente de Arganda & $13.2 \pm 0.0$ & $6.9 \pm 0.6$ & $7.9 \pm 0.0$ & $981 \pm 5$ & 1.59 & 14.1 & 23 \\
\hline 6 & $\begin{array}{l}\text { Manzanares (junta con } \\
\text { el río Jarama) }\end{array}$ & 12.9 & 1.5 & 7.5 & 953 & 5.63 & 34.6 & 93 \\
\hline 7 & $\begin{array}{l}\text { Jarama (junta con } \\
\text { el río Manzanares) }\end{array}$ & 8.9 & 4.8 & 7.7 & 1094 & 1.53 & 14.3 & 31 \\
\hline 9 & Presa del Rey & $11.7 \pm 0.0$ & $5.7 \pm 0.1$ & $8.0 \pm 0.0$ & $964 \pm 16$ & 3.67 & 25.1 & 60 \\
\hline 10 & Puente Pindoque & 15.3 & 4.5 & 7.7 & 942 & 3.81 & 23.2 & 51 \\
\hline 12 & $\begin{array}{c}\text { Puente hacia San Martín } \\
\text { de la Vega }\end{array}$ & $12.5 \pm 0.0$ & $7.0 \pm 0.0$ & $7.8 \pm 0.0$ & $939 \pm 31$ & 4.16 & 22.6 & 61 \\
\hline 5 & Laguna de El Campillo & $15.0 \pm 0.1$ & $6.3 \pm 0.2$ & $8.3 \pm 0.0$ & $1377 \pm 58$ & 0.20 & 1.6 & 38 \\
\hline 8 & $\begin{array}{l}\text { Laguna de Soto } \\
\text { de las Juntas }\end{array}$ & $14.3 \pm 0.0$ & $4.6 \pm 0.1$ & $7.5 \pm 0.1$ & $2307 \pm 12$ & 0.93 & 4.0 & 44 \\
\hline 11 & $\begin{array}{l}\text { Laguna de } \\
\text { Los Ángeles }\end{array}$ & $13.3 \pm 0.1$ & $7.5 \pm 0.9$ & $8.1 \pm 0.0$ & $1914 \pm 25$ & 0.08 & 8.7 & 12 \\
\hline 13 & $\begin{array}{l}\text { Laguna de Soto } \\
\text { Pajares } 5\end{array}$ & $14.7 \pm 0.1$ & $7.6 \pm 0.5$ & $8.8 \pm 0.0$ & $1297 \pm 62$ & 0.04 & 2.0 & 32 \\
\hline
\end{tabular}

Manzanares; se aprecia un claro incremento en las concentraciones de estos tres parámetros en las estaciones posteriores a la confluencia entre los ríos Manzanares y Jarama.

En la figura 2 y en la tabla 3 se muestran los resultados del Análisis de Componentes Principales efectuado sobre las variables analizadas en el agua de los ríos Jarama y Manzanares y de las lagunas de gravera adyacentes. El análisis explica el $67 \%$ del a varianza total en sus dos primeros factores. El primer componente principal (eje I, que explica un $45 \%$ de la varianza) está relacionado con la contaminación debida a la materia orgánica y a los nutrientes, y agrupa variables como la DQO, el nitrógeno total y el fósforo total en el extremo positivo del eje, en contraposición a la disponibilidad de oxígeno, en el extremo negativo del mismo. Sobre el eje II (que explica el $22 \%$ de la varianza), las variables de mayor peso son el cromo total y el cromo hexavalente. Otras variables, como el $\mathrm{pH}$, la temperatura y la conductividad, no alcanzan valores significativos

Tabla 3. Cargas de los factores en el Análisis de Componentes Principales para las variables estudiadas en el agua del río Jarama, el río Manzanares y las lagunas de gravera. Factor loadings in Principal Components Analysis of variables monitored in the water of the Rivers Jarama and Manzanares and in the gravel-pit lakes.

\begin{tabular}{lcc}
\hline VARIABLES & Factor I & Factor II \\
\hline Cromo hexavalente & 0.06 & 0.97 \\
Cromo total & 0.10 & 0.82 \\
DQO & 0.81 & 0.02 \\
Nitrógeno total & 0.89 & 0.33 \\
Fósforo total & 0.93 & 0.14 \\
Temperatura & -0.11 & -0.45 \\
Oxígeno disuelto & -0.82 & 0.23 \\
pH & -0.68 & -0.24 \\
Conductividad & -0.34 & -0.60 \\
\hline
\end{tabular}



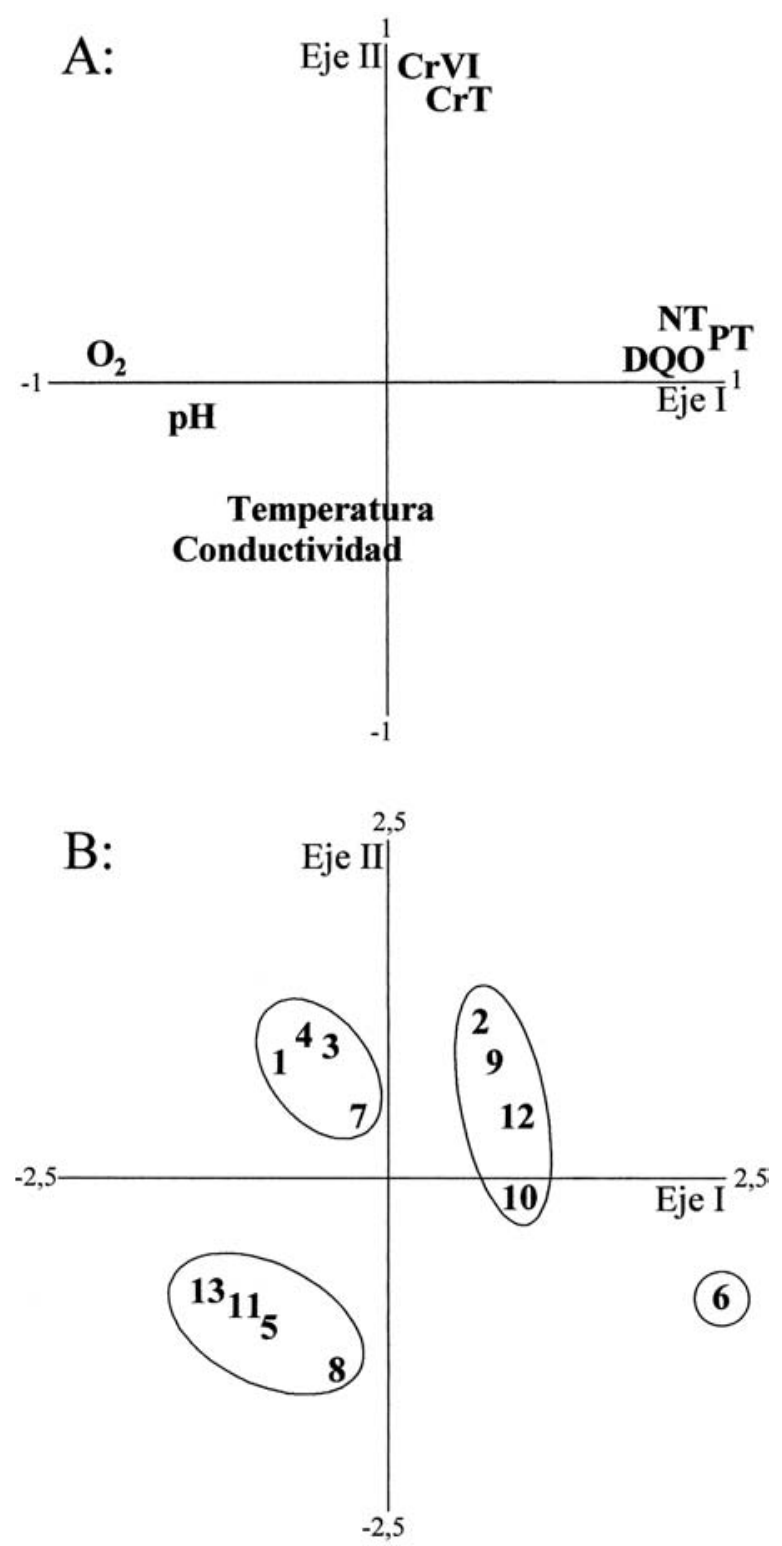

Figura 2. Análisis de Componentes Principales para las variables estudiadas en el agua de los ríos Jarama y Manzanares y en las lagunas de gravera (A). Proyección de los casos (estaciones de muestreo) sobre los componentes principales (B). Principal Components Analysis of variables monitored in the water of the Rivers Jarama and Manzanares and the gravel-pit lakes (A). Projection of the cases (sampling stations) on the principal components (B).

en los dos primeros componentes principales (Fig. 2, A). La proyección de las estaciones de muestro sobre los componentes I y II (Fig. 2, B) muestra una clara separación de la estación 6 (río Manzanares) con respecto a las demás. Este hecho se debe a la elevada carga de materia orgánica, a las altas concentraciones de nutrientes (procedentes de las aguas residuales urbanas de Madrid capital), a la menor disponibilidad de oxígeno disuelto (que es consumido en los intensos procesos de mineralización de la materia orgánica) y a la ausencia de contaminación por cromo en sus aguas. Otro grupo de clara distribución lo forman las estaciones 5, 8, 11 y 13, que corresponden a las cuatro lagunas de gravera (El Campillo, Soto de las Juntas, Los Ángeles y Soto de Pajares 5). En sus aguas no se observó contaminación por cromo y los niveles de nutrientes eran más bajos que los de los ríos Manzanares y Jarama. El tercer grupo lo integran las estaciones de muestro del Jarama previas a la desembocadura del Manzanares (estaciones $1,3,4$ y 7), con presencia de cromo, pero con DQO y nutrientes en concentraciones inferiores a las del grupo cuarto. En éste último, se agruparían las estaciones posteriores a la incorporación del Manzanares, junto con el efluente de la depuradora de Arganda (estaciones 2, 9, 10 y 12), todas ellas con niveles apreciables de cromo, con mayor carga orgánica y con elevadas concentraciones de nutrientes.

Respecto al muestreo en el medio intersticial (zona no inundada, a 1.5-2.0 $\mathrm{m}$ del cauce del río), se observaron concentraciones de cromo total y de cromo hexavalente extremadamente elevadas en el agua intersticial de las estaciones 3, 6, 7, 9 y 12 (Fig. 3), sobre todo en puntos como el Soto de El Rincón, la Presa del Rey y el Puente hacia San Martín de la Vega. A diferencia de lo observado en las muestras de agua superficial, la proporción de Cr VI con respecto al cromo total osciló entre el 0 y el $29 \%$. De acuerdo con los valores de $\mathrm{pH}$ (Tabla 4), la forma química predominante de Cr VI eran los cromatos (U.S.EPA, 1998). Las concentraciones de cromo total establecidas en los objetivos de calidad para aguas superficiales (Real Decreto 995/2000) se sobrepasaron entre uno y tres órdenes de magnitud; las establecidas para el $\mathrm{Cr}$ VI, entre uno y dos órdenes de magnitud. 


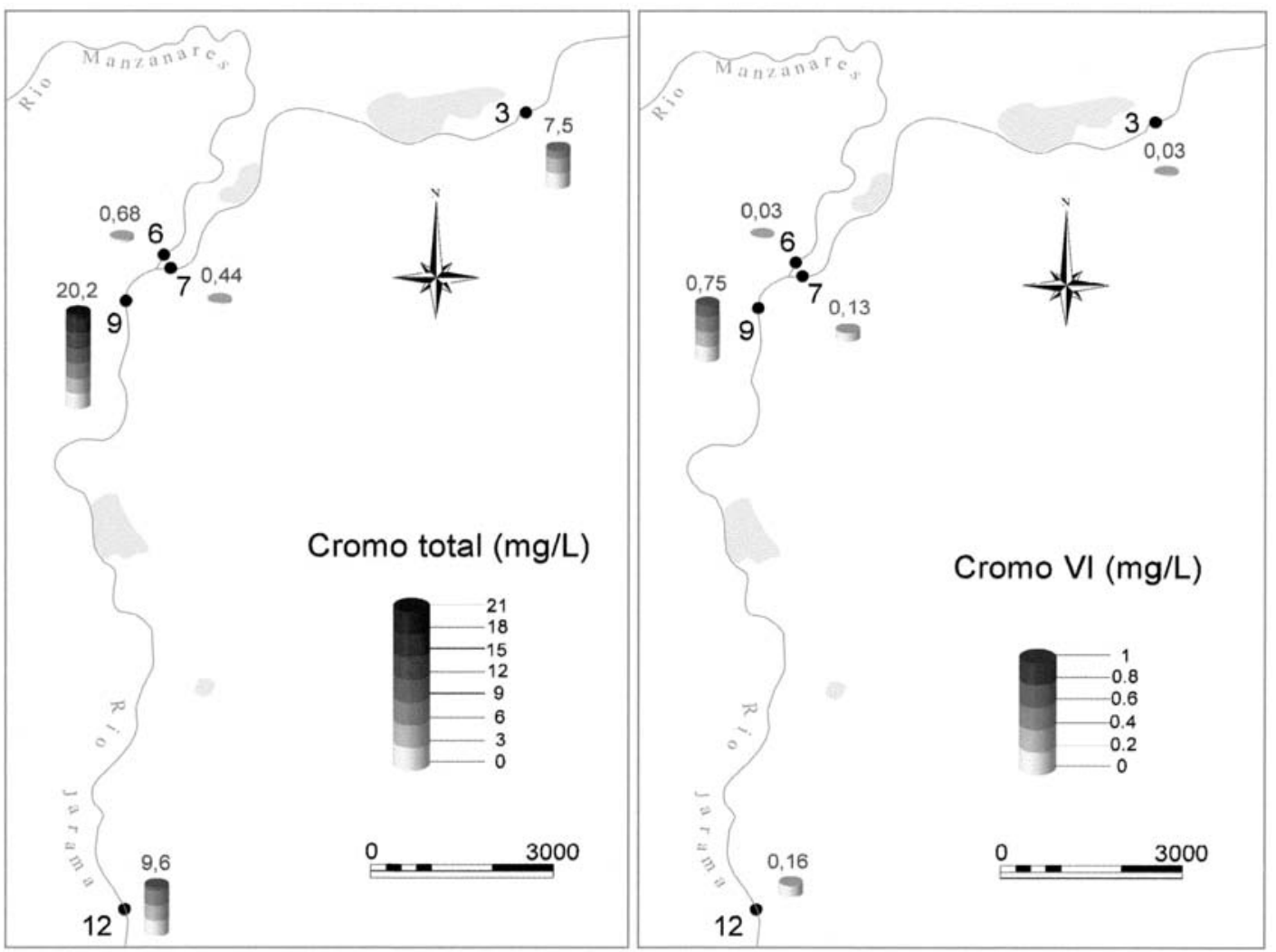

Figura 3. Concentraciones de cromo total y de cromo hexavalente en el agua intersticial. La numeración de las estaciones de muestreo corresponde a la descrita en la Tabla 1. Total chromium and hexavalent chromium concentrations in the interstitial water. Numbering of the sampling stations is shown in Table 1.

En la Tabla 4 se observa la escasa variabilidad de temperatura y $\mathrm{pH}$ en el agua intersticial entre estaciones. Durante el periodo en el que se realizaron los muestreos, el medio intersticial permanecía oxigenado, si bien la coloración negra de los

materiales más finos del suelo, observada durante la excavación (método de Karaman-Chappuis) en las estaciones $6,7,9$ y 12, sugería la existencia de periodos de anoxia en este medio. La conductividad decrecía siguiendo el curso del Jarama. Los

Tabla 4. Temperatura, oxígeno disuelto, $\mathrm{pH}$, conductividad, fósforo total, nitrógeno total y DQO en las muestras de agua intersticial. También se indica el "ángulo entre el litoral y el sentido de la corriente del río" para cada estación. Temperature, dissolved oxygen, pH, conductivity, total phosphorus, total nitrogen and COD in samples of interstitial water. "The angle between the river bank and the direction of the current "is also shown for each station.

\begin{tabular}{|c|c|c|c|c|c|c|c|c|c|}
\hline $\begin{array}{l}\text { Estación } \\
\left(\mathbf{n}^{\circ}\right)\end{array}$ & Localización & $\begin{array}{l}\text { Tra. } \\
\left({ }^{\circ} \mathrm{C}\right)\end{array}$ & $\underset{\left(\mathrm{mgL}^{-1}\right)}{\mathrm{O}_{2}}$ & $\begin{array}{c}\mathrm{pH} \\
\left(\mu \mathrm{Scm}^{-1}\right)\end{array}$ & $\begin{array}{l}\text { Cond. } \\
\left(\mathrm{mgL}^{-1}\right)\end{array}$ & $\underset{\left(\mathrm{mgL}^{-1}\right)}{\mathrm{PT}}$ & $\begin{array}{c}\mathrm{NT} \\
\left(\mathrm{mgL}^{-1}\right)\end{array}$ & $\begin{array}{c}\text { DQO } \\
\left({ }^{\circ}\right)\end{array}$ & Ángulo \\
\hline 3 & Soto de El Rincón & 12.6 & 6.4 & 7.6 & 1563 & 9.9 & 28.4 & 184 & 5 \\
\hline 6 & $\begin{array}{l}\text { Manzanares (junta con } \\
\text { el río Jarama) }\end{array}$ & 9.4 & 4.3 & 7.3 & 870 & 6.4 & 42.3 & 207 & 4 \\
\hline 7 & $\begin{array}{l}\text { Jarama (junta con } \\
\text { el río Manzanares) }\end{array}$ & 8.4 & 4.3 & 7.3 & 1048 & 5.4 & 32.5 & 138 & 4 \\
\hline 9 & Presa del Rey & 10.0 & 7.7 & 7.2 & 893 & 44.0 & 74.4 & 304 & 90 \\
\hline 12 & $\begin{array}{l}\text { Puente hacia San Martín } \\
\text { de la Vega }\end{array}$ & 10.1 & 8.0 & 7.5 & 792 & 23.9 & 42.0 & 334 & 90 \\
\hline
\end{tabular}



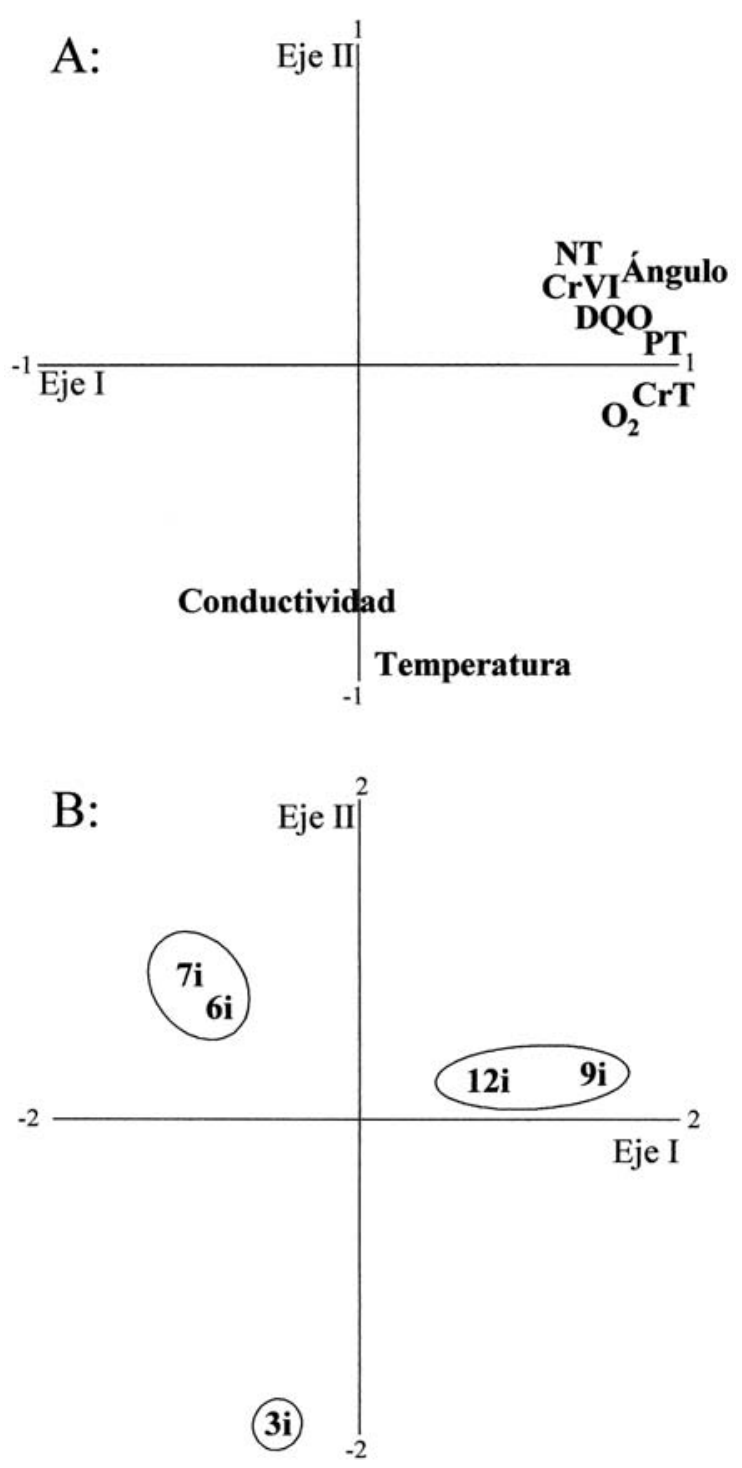

Figura 4. Análisis de Componentes Principales para las variables estudiadas en el agua intersticial (A). Proyección de los casos (estaciones de muestreo) sobre los componentes principales (B). Principal Components Analysis of variables monitored in the interstitial water (A). Projection of the cases (sampling stations) on the principal components $(B)$.

niveles de DQO, fósforo total y nitrógeno total resultaron muy elevados, siendo las estaciones 6 , 9 y 12 las más contaminadas.

En la figura 4 y en la Tabla 5 se muestran los resultados del Análisis de Componentes Principales efectuado sobre las variables analizadas en el agua intersticial. El análisis explica el $82 \%$ del a varianza total en sus dos primeros factores. El primer componente principal (eje I), que explica un $62 \%$ de la varianza, está relacionado con la contaminación (debida a la materia orgánica, el nitrógeno, el fósforo y el cromo). En este eje también tiene un peso importante la variable que hemos denominado "ángulo entre el litoral y el sentido de la corriente" (véase material y métodos). El eje II explica el 20\% de la varianza y agrupa a la temperatura y a la conductividad (figura 4, A). La proyección de las estaciones de muestro sobre los componentes I y II (figura 4, B) muestra un primer grupo formado por las estaciones 6 y 7 (confluencia de los ríos Manzanares y Jarama), donde se da de una zona común de influencia de ambos ríos sobre el medio intersticial; en ambas estaciones, la línea del litoral y el sentido de la corriente se situaban prácticamente en paralelo (Tabla 4) y los niveles de cromo, aunque elevados, eran inferiores a los del resto de los puntos de muestreo. En la estación 3 (Soto de El Rincón) se aprecia la influencia de la proximidad del efluente de la depuradora de Arganda; los niveles de nutrientes y de materia orgánica eran elevados, como en el grupo anterior, pero la concentración de cromo total era muy superior; en esta estación, el litoral y el sentido de la corriente se situaban también casi en paralelo (Tabla 4). El tercer grupo lo forman las estaciones 9 y 12 (Presa del Rey y Puente hacia San Martín de la Vega); en ambos puntos los niveles de contaminación eran muy elevados, tanto por la concentración de materia orgánica y de nutrientes, como por los niveles de cromo total y de cromo hexavalente; la disposición del litoral respecto al sentido de la corriente era perpendicular (Tabla 4).

En la figura 5 se presentan la concentración de cromo total en la fracción del sedimento de tamaño de partícula $<0.02 \mathrm{~mm}$ en todas las estaciones. Las muestras de sedimento fluvial presentaron concentraciones variables, dependiendo del punto de recogida. En la estación 1 (antes del vertido de la depuradora) se observó el valor más bajo ( $9 \mathrm{mg} \mathrm{kg}^{-1}$ de materia seca), mientras que en la estación 3 (salida de la depuradora al 
Tabla 5. Cargas de los factores en el Análisis de Componentes Principales para las variables estudiadas en el agua intersticial. Factor loadings in Principal Components Analysis of variables monitored in the interstitial water.

\begin{tabular}{lcc}
\hline Variables & Factor I & Factor II \\
\hline Cromo hexavalente & 0.77 & 0.24 \\
Cromo total & 0.94 & -0.15 \\
DQO & 0.83 & 0.16 \\
Nitrógeno total & 0.77 & 0.35 \\
Fósforo total & 0.96 & 0.11 \\
Temperatura & 0.18 & -0.95 \\
Oxígeno disuelto & 0.88 & -0.22 \\
Ángulo & 0.89 & 0.21 \\
Conductividad & -0.36 & -0.85 \\
\hline
\end{tabular}

río Jarama) se dio la concentración más elevada (5128 $\mathrm{mg} \mathrm{kg}^{-1}$ de materia seca). En el resto de las estaciones fluviales los niveles de cromo se mantuvieron entre 60 y $250 \mathrm{mg} \mathrm{kg}^{-1}$ de materia seca. Las concentraciones de cromo en el sedimento de las lagunas de gravera no superaron los $2 \mathrm{mg} \mathrm{kg}^{-1}$ de materia seca.

\section{DISCUSIÓN}

Margalef (1983) estimó un rango para la concentración de cromo en las aguas naturales epicontinentales entre 0.1 y $1.4 \mu \mathrm{g} \mathrm{L}-1$. U.S.EPA (1984) citó concentraciones de cromo total en los ríos Amazonas y Yukon de 2 y $2.3 \mu \mathrm{g} \mathrm{L}^{-1}$, respectivamente. Friederich \& Müller (1984) citaron una concertación media de cromo de $7.9 \mu \mathrm{g} \mathrm{L}^{-1}$ en el tramo bajo del Rin. Pettine el al. (1992) observaron concentraciones medias de cromo en el río Po de $9.3 \mu \mathrm{g} \mathrm{L}^{-1}$. En los lagos Hurón y Superior las concentraciones medias de cromo eran de $0.4 \mu \mathrm{g} \mathrm{L}^{-1}$, mientras que en su ríos tributarios, Ontonagon, Carp River y Saginaw River, se daban valores de $11,3.3$ y $6.5 \mu \mathrm{g} \mathrm{L}^{-1}$, respectivamente (Poldoski et al., 1978). Las concentraciones observadas en el río Jarama superaron ampliamente tales valores de referencia.

Uno de los ecosistemas en los que cabría esperar la acumulación del contaminante es el constituido por los campos de cultivo que reciben siste-

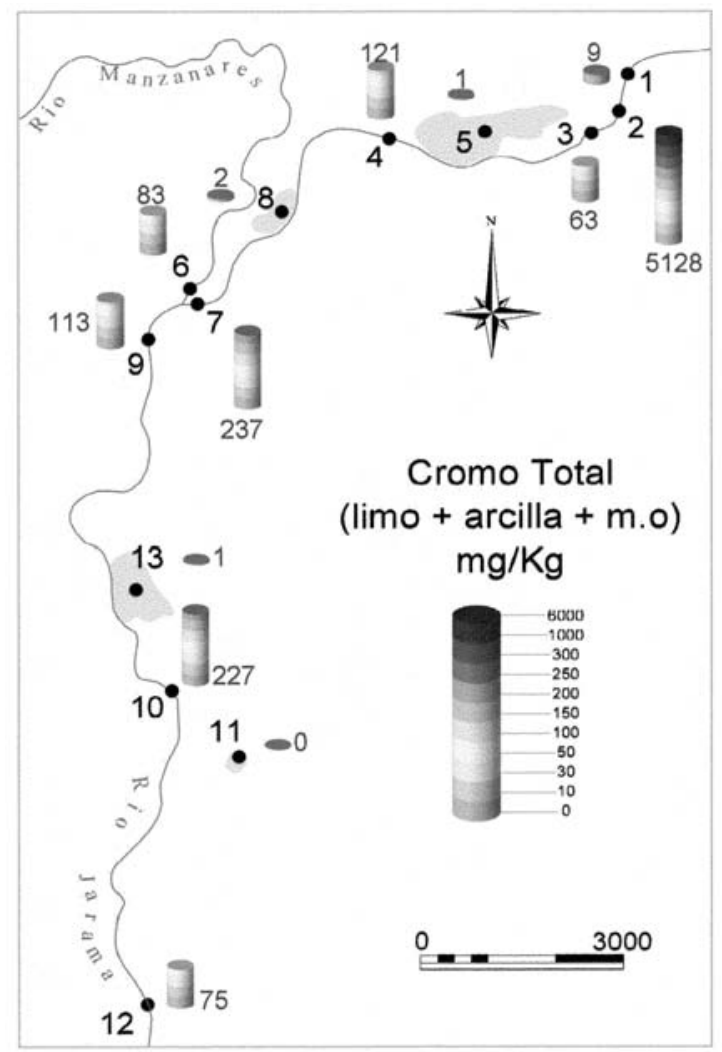

Figura 5. Concentración de cromo total en la fracción del sedimento de tamaño de partícula $<0.02 \mathrm{~mm}$, en las estaciones de muestro de los ríos Jarama y Manzanares y en las lagunas de gravera. La numeración de las estaciones corresponde a la descrita en la Tabla 1. Total chromium concentration in sediments (fraction $<0.02 \mathrm{~mm}$ ) of the Rivers Jarama and Manzanares and in the gravel-pit lakes. Numbering of each sampling station is shown in Table 1.

máticamente aguas de riego que exceden los niveles permisibles de cromo. En la Presa del Rey (estación 9) se realiza la toma de agua para abastecer la Real Acequia del Jarama, que suministra agua de riego a la zona hortícola comprendida entre el término municipal de San Martín de la Vega y la confluencia de los ríos Jarama y Tajo. Estudios previos de Cala \& Rodríguez (1983) mostraron un valor medio anual de $0.15 \pm 0.14 \mathrm{mg} \mathrm{L}^{-1}$ de cromo total (media \pm desviación estándar) en las aguas de la Real Acequia del Jarama y de $0.12 \pm 0.13 \mathrm{mg} \mathrm{L}^{-1}$ en las aguas del Jarama (en estaciones próximas a la localidad de Titulcia, ligeramente más al sur de nuestro área de trabajo). Veinte años más tarde, se cons- 
tata que el orden de magnitud en la concentración de cromo no ha variado con respecto a los valores observados entonces. Asimismo, en su estudio sobre la contaminación por metales pesados en los suelos de la Vega de Aranjuez, Cala et al. (1985) observaron elevados niveles de contaminación por cromo, níquel, zinc, cobre, plomo y cadmio en suelos situados a la orilla del Jarama (a la altura de Titulcia). Aunque no existe bibliografía sobre los niveles actuales de contaminación de los suelos agrícolas por metales en la zona de estudio, cabe esperar que se haya producido un incremento, dado el proceso acumulativo por la práctica continuada de riegos con aguas que exceden los niveles admisibles. Respecto a la posible existencia de procesos de contaminación de las aguas freáticas por lixiviación de los metales pesados desde los suelos de cultivo contaminados, diversas investigaciones sobre el movimiento de los metales pesados en el suelo han concluido que la mayoría de los mismos tienden a permanecer en superficie (Sidle et al., 1976; Parker et al., 1978). Parece improbable, por tanto, que los procesos de lixiviación en las zonas de regadío contribuyan a la contaminación del acuífero subyacente. Tampoco se han observado filtraciones del contaminante desde el río hacia las lagunas de gravera adyacentes (Figs. 1 y 5); no puede decirse, a la vista de los resultados, que las aguas subterráneas a ambos lados del cauce principal se vean afectadas por un proceso de dispersión del cromo. Sin embargo, el medio intersticial y los sedimentos del río aparecen fuertemente contaminados, constituyendo importantes reservorios de cromo que pueden actuar como fuentes potenciales para la dispersión del mismo, hacia las aguas del propio cauce del río: cambios ambientales hacia condiciones oxidantes y $\mathrm{pH}$ elevado podrían favorecer la oxidación del cromo trivalente a hexavalente, desencadenando su movilización hacia la fase disuelta. Pettine et al. (1991) han investigado la cinética de oxidación del cromo trivalente, estimando un tiempo medio de 45 días para su oxidación en aguas marinas, a $\mathrm{pH} 8$ y $25^{\circ} \mathrm{C}$.

Se ha observado una evidente influencia de la morfología del cauce sobre los procesos de acu- mulación de contaminantes en el medio intersticial. En las zonas del litoral que oponen resistencia a la corriente del agua las concentraciones de contaminantes son más elevadas, debido al efecto de filtrado y acumulación: el cromo transportado por la corriente es fácilmente adsorbido por la abundante presencia de limos y materia orgánica acumulados en el medio intersticial, en el que, además, se favorece la acumulación de las partículas en suspensión transportadas por el río, con cromo ya adsorbido. Respecto a los posibles efectos de la abundancia de cromo sobre la fauna intersticial, Del Valls et al. (1997) determinaron, mediante ensayos toxicológicos sobre Brachionus plicatilis, que sólo a concentraciones de cromo iguales o inferiores a $0.0045 \mathrm{mg} \mathrm{L}^{-1}$ no se observaban efectos adversos sobre la comunidad biológica.

En las aguas del río Jarama, la mayor parte del cromo se encontraba en forma hexavalente. Durante la época del año en la que se realizó la campaña de muestreos (noviembre de 2001) no se observaron condiciones reductoras que pudiesen favorecer el proceso de reducción de cromo VI a cromo III. Sin embargo, los elevados niveles observados de DQO sugieren el desarrollo de intensos procesos de mineralización de la materia orgánica y, por tanto, de consumo de oxígeno, durante periodos más cálidos. Tras el proceso de reducción, la solubilidad de los compuestos de cromo trivalente quedaría entonces limitada por la formación de diversos óxidos e hidróxidos de cromo, que precipitan en un rango de pH 5-12 (Katz \& Salem, 1994).

La toxicidad crónica del cromo hexavalente para los peces ha sido estudiada por Olson (1958, en: U.S. EPA, 1972), Olson \& Foster (1956, 1957, en: U.S. EPA, 1972), Doudorof \& Katz (1953, en: U.S. EPA, 1972) y Cairns (1956, en: U.S. EPA, 1972), sin embargo, los datos son todavía incompletos. Para salmónidos se recomiendan concentraciones de cromo total inferiores a $0,02 \mathrm{mg} \mathrm{L}^{-1}$ (U.S. EPA, 1972). Gagneten (2002) ha observado que la recuperación de comunidades zooplanctónicas expuestas a diversas concentraciones de cromo, sólo es posible a concentraciones inferiores a $0.1 \mathrm{mg} \mathrm{L}^{-1}$; la den- 
sidad, la riqueza de especies y la diversidad disminuyen significativamente con el incremento en la concentración de cromo.

Por otra parte, la elevada concentración de cromo hexavalente en las aguas del Jarama entraña un importante riesgo para la salud humana debido a su elevada carcinogenicidad por inhalación (U.S. EPA, 1998). Teniendo en cuenta que estas aguas se vienen utilizando tradicionalmente para riego agrícola, conviene desaconsejar el riego por aspersión para evitar la inhalación de aerosoles contaminados por Cr VI. U.S. EPA (1998) sitúa el umbral de riesgo por inhalación en $1.2 \times 10^{-2} \mu \mathrm{g} \mathrm{m}^{-3}$ de Cromo VI en aire.

El análisis de los sedimentos brinda un excelente panorama del grado de contaminación dentro de un área determinada (Bryan y Langston, 1992). Rovira (1993) describió un enriquecimiento generalizado en metales pesados en los sedimentos del río Jarama debido a causas antropogénicas, clasificando los sedimentos de las estaciones de Puente de Arganda y Presa del Rey como fuertemente contaminados por cromo. International Joint Commission (1982), en su clasificación de los sedimentos de las bahías de los Grandes Lagos de EEUU, considera sedimentos naturales no contaminados por cromo a aquellos con concentraciones por debajo de $25 \mathrm{mg} \mathrm{kg}^{-1}$, medianamente contaminados a los que se encuentran en el rango entre 25 y $75 \mathrm{mg} \mathrm{kg}^{-1}$ y fuertemente contaminados a los de concentraciones superiores a $75 \mathrm{mg} \mathrm{kg}^{-1}$. Las muestras de sedimentos fluviales recogidas en el presente estudio superaron el umbral de los $75 \mathrm{mg} \mathrm{kg}^{-1}$ en las estaciones 2, 4, 6, 7, 9, 10 y 12. La mayor parte del cromo de los sedimentos acuáticos se encuentra en la forma trivalente $\mathrm{y}$, a pesar de no ser tan móvil en el medio ambiente como el cromo hexavalente, se ha demostrado experimentalmente su acumulación y movimiento hacia los eslabones superiores de la cadena trófica (Aislabie \& Loutit, 1986, Bremer \& Loutit, 1986). En el caso de los peces de agua dulce y de los crustáceos, la absorción de metales a través de los alimentos puede ser muy significativa (Heath, 1987; Dallinger et al., 1987). La absorción por las plantas puede cons- tituir, asimismo, una vía importante para que los metales presentes en los sedimentos se encuentren biodisponibles para las especies herbívoras (Berg et al., 1995).

\section{BIBLIOGRAFÍA}

AISLABIE, J. \& M. W. LOUTIT. 1986. Accumulation of Cr III by bacteria isolated from polluted sediment. Marine Environ. Res., 20: 221-232.

APHA. 1989. Standard methods for the examination of water and wastewater. American Public Health Association. Washington DC.

ARAUZO, M., M. VALLADOLID, C. NOREÑA \& O. CEDENILLA. 2002. Limnología y contaminación: el lagunaje profundo como tratamiento ecológico de las aguas residuales urbanas y mixtas. En: Ciencia y Medio ambiente. Segundas Jornadas Cientificas sobre Medio ambiente del CCMACSIC. F. Valladares, (ed.): 165-174. Centro de Ciencias Medioambientales (CSIC), Ministerio de Ciencia y Tecnología, Madrid.

BARTLETT, R. J. \& J. M. KIMBLE. 1976. Behaviour of chromium in soils. II. Hexavalent forms. $J$. Environ. Qual., 5: 383-386.

BERG, H., M. KIIBUS \& N. KAUTSKY. 1995. Heavy metals in tropical Lake Kariba, Zimbabwe. Wat. Air and Soil Poll., 83:237-252.

BOUYOUCOS, 1929. A new simple and rapid method for determining the moisture equivalent of soil and the role of soil colloid on this moisture equivalent. Soil Sci., 27 (3).

BREMER, P. J, \& M. W. LOUTIT. 1986. Bacterial polysaccharide as a vehicle for entry of $\mathrm{Cr}$ (III) to a food chain. Marine environ. Res., 20: 235-248.

BRYAN, G. W. \& W. J. LANGSTON. 1992. Bioavailability $\mathrm{m}$ accumulation and effects of heavy metals in sediments with special reference to United Kingdom Estuaries: a review. Environ. Poll., 76: 89-131.

CALA, V. \& J. RODRÍGUEZ. 1983. Distribución zonal y estacional de metales pesados en las aguas de confluencia de los ríos Jarama y Tajo. Actas del V Congreso Nacional de Química, Puerto de la Cruz, España: 443-451.

CALA. V., J. RODRÍGUEZ \& A. GERRA. 1985. Contaminación por metales pesados en los suelos de la Vega de Aranjuez. (I) $\mathrm{Pb}, \mathrm{Cd}, \mathrm{Cu}, \mathrm{Zn}, \mathrm{Ni}$ y $\mathrm{Cr}$. An. Edafol. y Agrobiol., 44: 1595-1608. 
CHAPPUIS, P. A. 1942. Eine neue methode zur Untersuchung der Grundwasserfauna. Acta Sc. Math. Nat. Universität Franz Josepfine, 6: 1-7.

DANLligER, R., F. PROSI, H. SENGER \& H. BACK. 1987. Contaminated food and uptake of heavy metals by fish. A review and proposal for further research. Oecologia, 73: 91-98.

DEL VALLS, T. A., L. M. LUBIÁN, J. M. FORJA \& A. GÓMEZ-PARRA. 1997. Comparative ecotoxicity of interstitial waters in littoral ecosystems using Microtox and the rotifer Brachionus plicatilis. Environ. Toxicol. Chem., 16: 2333-2338.

EARY, L. E. \& D. RAI. 1989. Kinetics of chromate reduction by ferrous ions derived from hematite and biotite at $25^{\circ}$ C. Am. J. Sci., 289: 180-213.

FÖRSTNER, U. 1983. Assessment of metal pollution in rivers and estuaries. En: Applied Environmental Geochemistry. I. Thorton (ed.). Academic Press, London.

FÖRSTNER, U. \& G. T. W. WITTMANN. 1981. Metal pollution in the aquatic environment. Springer-Verlag. Berlin.

FRIEDRICH, G. \& D. MÜLLER. 1984. Rhine. En: Ecology of European Rivers. M.A. Whitton (ed.): 265-315. Blackwell Scientific Publications. Oxford.

GAGNETEN, A. M. 2002. Respuesta de la comunidad zooplanctónica de agua dulce a la aplicación de cromo en clausuras experimentales. Interciencia, 27(10): 563-570.

HEATH, A. G. 1987. The effects of waterborne copper or zinc on the osmoregularity response of bluegill to a hypertonic $\mathrm{NaCl}$ challenge. Comparative Biochem. And Physiol., 88: 307-311.

INTERNATIONAL JOINT COMMISSION (IJC). 1982. Guidelines and Register for Evaluation of Great Lakes Dredging Projects. Report of the Dredging Subcommittee to the Water Quality Programs Committee of the Great Lakes Water Quality Board. Windsor, Ontario.

KATZ, S. A. \& H. SALEM. 1994. The biological and environmental chemistry of chromium. VCH, New York.

LANGE, 1998. Handbook of photometrical operation analysis. LASA/IP/CADAS/ISIS. Great Britain.

MARGALEF, R. 1983. Limnología. Omega, Barcelona.

MORIARTY, F. \& H. M. HANSON. 1988. Heavy metals in sediments of the River Ecclesbourne, Derbyshire. Water Res., 22: 475-480.

MURPHY, J. \& J. P. RILEY. 1962. A modified single solution method for the determination of phosphate in natural waters. Anal. Chim. Acta, 27: 31-36.
PALMER, S. A. K., M. A. BERTON, T. J. NUNNO, D. M. SULLIVAN \& N. F. SURPRENANT. 1988. Metal/cyanide containing wastes: treatment technologies. Pollution Technology Review $\mathrm{N}^{\circ} 158$. Noyes Data Corporation, New Jersey.

PARKER, G. R., W. W. McFEE \& J. M. KELLY. 1978. Metal distribution in forested ecosystems in urban and rural northwested Indiana. J. Envir. Qual., 7: 337-342.

PETTINE, M., F. J. MIRELLO \& T. L. NOCE. 1991. Chromium (III) interactions in seawater through its oxidation kinetics. Mar. Chem, 34: 24-46.

PETTINE, M., M. CAMUSSO \& W. MARTINOTTI. 1992. Dissolved and particulate transport of arsenic and chromium in the River Po. Sci. Total. Environ., 119: 253-280.

POLDOSKI, J. E., E. N. LEONARD, J. FIANDT, L. ANDERSON, G. OLSON \& G. GLASS. 1978. Factor in the determination of selected trace elements in near-shore U.S. waters of Lake superior and Lake Huron. J. Int. Assoc. Grat Lakes Res., 4: 206-215.

ROVIRA, J. V. 1993. Contaminación por metales pesados en los sedimentos del río Jarama y su bioasimilación en Tubifícidos. Tesis doctoral. Universidad Complutense. Madrid.

SALEH, F. Y., T. F. PARKERTON, R. V. LEWIS, J. H. HUANG \& K. L. DICKSON. 1989. Kinetics of chromium transformation in the environment. Sci. Total Environ., 86: 25-41.

SIDLE, R. C., J. E. HOOK \& L. T. KARDOS. 1976. Heavy metal application and plant uptake in a land disposal system for wastewater. J. Environ. Qual., 5: 97-102.

STATSOFT, INC. STAFF. 1995. Quick Statistica for Windows. Statsoft Incorporated. Tulsa.

U.S. EPA. 1972. Water quality criteria. Federal Water Pollution Control Administration. Washington, DC.

U. S. EPA, 1984. Health assessment document for chromium. EPA-600/8-83-014F. U.S. Environmental Protection Agency, Research Triangle Park, N.C.

U.S. EPA. 1992. Manual of guidelines for water reuse. U.S. Environmental Protection Agency 625/R92/004. Center of Environmental Research Information. Cincinnati.

U.S. EPA. 1998. Toxicological review of hexavalente chromium. U.S. Environmental Protection Agency 18549-29-9. Washington DC.

W.A. EPA. 1993. Western Australian Quality Guidelines for Fresh and Marine Water. EPA Bulletin $\mathrm{n}^{\circ}$ 711. Western Australian Environmental Protection Authority, Perth. 